\title{
Mitteilungen des Magnesiumforum Bad Radkersburg
}

J. Miner. Stoffwechs. Muskuloskelet. Erkrank. 2019 • 26:24

https://doi.org/10.1007/s41970-019-0061-x

(c) Springer-Verlag GmbH Austria, ein Teil von Springer Nature 2019
Ärztliche Arbeitsgemeinschaft für Lebensstilmedizin

p.a. Prim. Dr. Bernd ZIRM (V.i.S.d.P.)

8490 Bad Radkersburg -

Vita med Gesundheitszentrum, Alfred-Merlini-Straße 7

Tel.: 03476/3403

Fax: 03476/3403

Homepage: www.lebensstil-medizin.at

E-Mail: bernd.zirm@gmx.at
Liebe Kolleginnen!

Liebe Kollegen!

Magnesium hat wieder einmal das Interesse von Forschern und Anwendern geweckt. Nach einer Zeit, in der man den Slogan „Nutzt es nichts, so schadet es nicht" verwendet hat, zeigt sich nun, dass viele Autoren Magnesium als einen wesentlichen Risikofaktor für Herz-Kreislauf-Erkrankungen, Herzrhythmusstörungen und der Entstehung von Diabetes mellitus Typ II sehen.

Vielleicht hat auch die Industrie das ihre dazu beigetragen, indem sie Darreichungsformen jeder Art anbietet und das wichtige Elektrolyt Kalium mitverarbeitet, da Kalium ein Schlüssel-Ion für die Zelle darstellt.

Auch Mineralwasserhersteller, insbesondere die Wasser- aufbereiter mit ihrer „rosa Werbung", sind aktiv und bieten Trinkwasser mit Magnesium versetzt für den Alltag an.

Zurzeit starten wir in Bad Radkersburg eine Testserie, bei der wir freiwillige Probanden ersuchen, eine definierte Magnesiummenge entweder auf einmal oder über den Tag verteilt zuzuführen. Der Vergleich wird im Wohlbefinden (Magen-DarmProbleme) sowie im Blutspiegel bei jedem Probanden gemessen. Die Ergebnisse sollten im Herbst 2019 vorliegen.

Ich darf Ihnen eine schöne Sommerzeit wünschen.

Mit freundlichen Grüßen aus der Südoststeiermark!

Prim. Dr. Bernd Zirm und sein Team

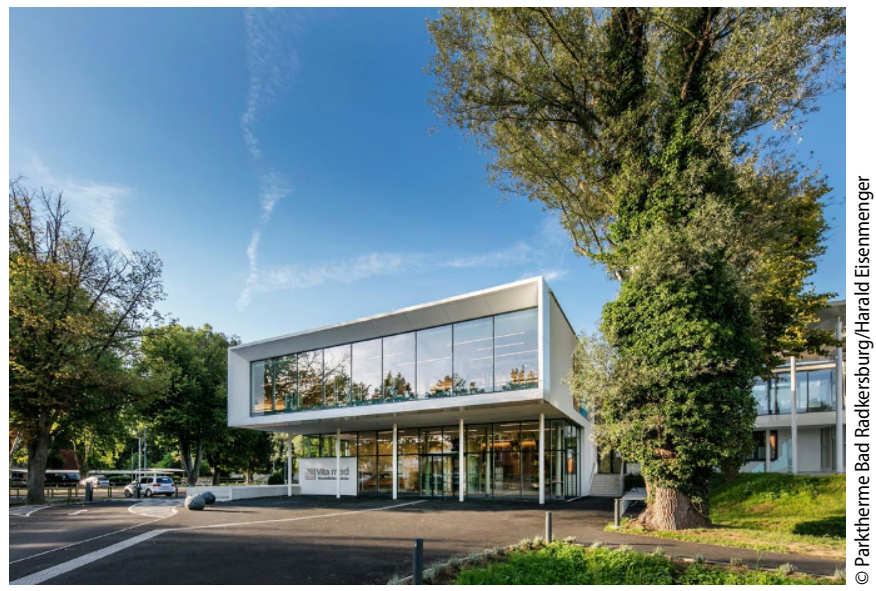

Hier steht eine Anzeige. 